\section{Die wichtigsten Fortschritte auf dem Gebiete der anorg. Großindustrie im Jahre 1908.}

\author{
Von Dr. H. v. KÉLER.
}

(Vortrag, gehalten auf der Wanderversammlung des Vereins Deutscher Chemiker in Kobln am 27./3. 1909.)

(Eingeg. 19./4. 1909.)

Die anorganisch-chemische Großindustrie hatte auch im verflossenen Jahre manchen Fortschritt zu verzeichnen. Der besseren Utbersicht wegen teile ich das ganze Gebiet der anorganischen Großindustrie in Gruppen und beginne mit der

Industrie des Schwefels.

Mit dem steten Anwachsen der amerikanisehen Schwefelgewinnung steigert sich die Schwierigkeit einer rentablen Verarbeitung des sizilianischen Rohschwefels. Die Vorräte mehren sich und sollen bereits Ende Oktober vorigen Jahres auf 556 I57 t angewachsen sein (Dr. K. $R \mathbf{e u} \mathrm{s} \mathbf{h}$, Jahresberichte über die Industrie der Mineralsäuren und des Chlorkalkes, Chem.-Ztg. S. 190); auch sollen die Verschiffungen eher ab- als zunehmen.

Unter den Vorschlägen zur Beseitigung dieser Krisis ist der zur Verwertung des Rohschwefels für die Schwefelsäurefabrikation hervorzuheben. Um aber eine Konkurrenz mit den Pyriten zu ermöglichen, müßten vor allem die Transportverhältnisse von den Gruben zur Küste verbessert, Ausnahmetarife für Schwefel eingeführt und die Verschiffungsgelegenheiten entsprechend umgestaitet werden. Und so ist man zunächst bestrebt, durch Verbesserungen in Konstruktion der Sahwefelöfen der bedrohten Industrie zu helfen.

Arthur Walter in Neapel1) will Blockschwefel oder Blüte aus dem $20-40 \%$ Rohschwefel gewinnen, ohne ihn vorher in meilerartigen Haufen (Calcarone) oder den gemauerten Öfen, System Gil1, auszuschmelzen.

Durch einen schrägen, drehbaren eisernen $\mathbf{Z y -}$ linder werden inerte Gase, die auf $150-200^{\circ}$ erhitzt sind, geblasen. Das Schwefelerz wird durch eine Schnecke kontinuierlich dem Zylinder zugeführt und gleitet in ihm herunter. Der Schwefel schmilzt und wird an der tiefsten Stelle abgezogen, das entschwefelte Erz kontinuierlich entfernt.

Ferner hat $W$ a lte $r^{2}$ ) einen Röstofen kon: struiert in dem ein Teil des Schwefels als solcher, ein Teil in Form von $\mathrm{SO}_{2}$ gewonnen wird. Es ist ein gewöhnlicher Etagenofen, nur mit der $\mathrm{Ab}$ änderung, daß die Röstgase unterhalb der beiden obersten Etagen seitlich abgeführt werden. Es wird dadurch im oberen Teile des Ofens ein von Röstluft freier Schmelzraum geschaffen, aus dem der $S$ nach außen abgeführt wird. Das von Schwefel teilweise befreite Material wird dann auf den unteren Platten ganz abgeröstet.

Ein anderer Schwefelofen wird von der $S$ a chsenburger-Aktienmaschinenfabrikund EisengieBerei Sachsen. burg-Heldungen ${ }^{3}$ ) gebaut. Es ist dies

\footnotetext{
1) Nr. 192472 ; diese Z. 21, 792 (1908).

2) Nr. 192 518; diese Z. 21, 792 (1908).

3) Nr. 196 371; diese Z. 21, 1563 (1908).
}

ein Muffelofen mit eigenartiger Zuführung des Schwefelerzes. Auf der Sohle der Muffel stehen mehrere tönerne, flache Schalen. Ùber jeder Schale befindet sich in der Decke der Muffel ein Fülltrichter, der in ein gußeisernes Rohr nach unten zuläuft. Dieses Rohr reicht bis in das Innere der Schale in den geschmolzenen Schwefel hinein, so daß ein gasdichter $A$ bschluß vorhanden ist.

Ist der Ofen heiß, so kommt der $S$ in den Füllrohren zum Schmelzen, läuft in die darunter stehende Schale und über deren Rand auf die Sole der Muffel, von wo er kontinuierlich abgezogen wird.

Durch diese Einrichtung soll ein Zusammenbacken des Materials in den Einfüllschächten vermieden werden.

Hippolyt Köhler-Berlin ${ }^{4}$ ) gewinnt feinstverteilten Schwefel dadurch, daß er ein Gemenge von gleichen Teilen Schwefel und Naphthalin schmilzt und die Schmelze erkalten läßt. Beim Abkühlen bilden sich Krystalle, die nicht aus reinem $\mathrm{S}$ und Naphthalin zusammengesetzt sind, so daß hier eine sogenannte feste Lösung vorliegt. Aus dieser Masse wird nach Entfernung des Naph. thalins, die durch mäBiges Erwärmen in einem Gasstrom oder durch Extraktion mit einem geeigneten Lösungsmittel erfolgt, der Schwefel in fein verteiltem Zustand erhalten.

Von sonstigen neuen Fabrikationsverfahren von Schwefel wären die Gewinnung aus $\mathrm{SO}_{2}$ und $\mathrm{CO}$ und die aus $\mathrm{SO}_{2}$ und $\mathrm{H}_{2} \mathrm{~S}$ zu erwähnen.

Die Soeiété anonyme metal. I r r ique Procédés de Laval-Brüssel5) gewinnt Schwefel aus $\mathrm{SO}_{2}$ und $\mathrm{CO}$. An und für sich ist die dem Verfahren zugrunde liegende Reaktion bekannt. Sie wurde aber bisher nicht praktisch arssgeführt, weil es nur unvollständig gelang, die beiden Gase miteinander zu mischen. Eine auBerordentlich innige Mischung wollen nun die Erfinder dadurch erreichen, daB das $\mathrm{CO}$ in das betreffende Mischgefä $B$ tangential, das $\mathrm{SO}_{2}$ gleichzeitig zentral eingeführt wird. Dadurch wird der $\mathrm{SO}_{2}$-Strom in Umdrehung versetzt, mischt sich innig mit dem $\mathrm{CO}$, und die sich bildenden S-haltigen Dämpfe werden mit dem Gasstrom fortgeführt.

Die Gewinnung von $\mathrm{S}$ aus $\mathrm{SO}_{2}$ und $\mathrm{H}_{2} \mathrm{~S}$, die $\operatorname{sich} W$ alter Feld in $Z$ eh-lend orf $\mathbf{f}^{6}$ ) hat patentieren lassen, beruht auf der Gleichung

$$
2 \mathrm{H}_{2} \mathrm{~S}+\mathrm{SO}_{2}=3 \mathrm{~S}+\mathrm{H}_{2} \mathrm{O} \text {. }
$$

Die Umsetzung verläuft nur unvollständig und wird durch die Bildung von Polythionsäuren gestört. Durch Einleiten der Gase in $\mathrm{MgCl}_{2}$ versuchte man, diesem U̇belstande abzuhelfen, doch ohne Erfolg. Wa 1 t e r F e ld leitet nun das Gasgemisch in Flüssigkeiten ein, die den entstehenden Schwefel zu lösen vermögen. Hierzu eignen sich am besten die Schweröle der Teerdestillation. Die Öle erwärmen sich dabei, was die Lösungsfähigkeit für den S erhöht. Da der entstehende Schwefel sich sofort in den Ölen löst, wird verhindert, daß er mit dem gleichzeitig entstehenden $\mathrm{H}_{2} \mathrm{O}$ Thiosulfate und Polythionsäuren bildet.
4) Nr. 192815 ; diese Z. 21, 792 (1908).
5) Nr. 196371 ; diese Z. 21, 1563 (1908).
6) Nr. 202 349; diese Z. 21, 2375 (1908). 
Schwefelsäure nach dem Kammer. verfahren.

Die alte Schwefelsäurefabrikation nach dem Kammerverfahren ist fortwährend auf das eifrigste bemüht, durch Verbesserungen, die auf eine möglichst intensive Ausnutzung des Kammerraumes, eine Herabdrückung der Gestehungskosten durch Ersatz des Dampfes durch $\mathrm{H}_{2} \mathrm{O}$ hinzielen, erfolgreich mit dem Kontaktprozeß in Konkurrenz zu treten. So sind denn auch im vergangenen Jahre hier zahlreiche Verbesserungen patentiert worden.

Was die Öfen zur Erzeugung der $\mathrm{SO}_{2}$ betrifft, so sind, um mit den, ,S c h w e f e l" Ö f e n zu beginnen, zwei neue Röstöfen für Schwefel konstruiert worden, ein Ofen von Thomas Adam C l a y ton und ein Ofen von der $\mathrm{ch}$ e $\mathrm{m}$ is $\mathrm{e} \mathrm{h}$ en Fabrik auf Aktien vorm. Schering ${ }^{7}$ ). Beim Clayt o n schen Ofen wird die zur Verbrennung des Schwefels nötige Luftmenge selbsttätig reguliert und zwar unter Benutzung der Differenzialbewegung zwischen einem erhitzten und einem kalten Rohre.

In dem Schwefelofen ist ein Rohr angeordnet, welches entweder innerhalb der S-Charge oder zweckmäBig in einiger Entfernung über dieser angebracht ist. Das eine Ende des Rohres ist mit der einen Ofenwandung fest verbunden, während das andere sich durch die entgegengesetzte Wandung des Ofens frei hindurch bewegen kann und einen Zapfen trägt, an welchem ein Hebel angreift. Innerhalb dieses Rohres ist ein zweites angeordnet, welches mit dem ersten durch einen Zapfen verbunden ist. Das innere Rohr wird durch einen $\mathrm{H}_{2} \mathrm{O}$-Strom kalt gehalten. Wenn das äußere Rohr durch die Röstgase heiß wird, dehnt es sich aus, während das innere Rohr annähernd seine ursprüngliche Länge behält. Die durch die Ausdehnung des äuBeren Rohres verursachte Bewegung wird auf einen Hebel übertragen, welcher auf das Lufteinlaßventil einwirkt.

Infolgedessen wird, wenn die Temperatur des Ofens auf eine bestimmte Höhe steigt, Luft in den oberen Teil des Ofens einströmen können.

Bei dem Ofen von S chering ${ }^{8}$ ) wird feinkörniger oder pulverförmiger Schwefel mit einem Strom von Luft und Sauerstoff verbrannt. Bei Inbetriebsetzung des Ofens wird der Schwefel in dem Gasstrom durch eine Flamme entzündet. Der weitere Zufluß des Schwefels wird so reguliert, daß der Schwefel in dem Momente des Zusammentreffens mit dem Luft- oder Sauerstoffstrom sich en tzündet und sofort verbrennt.

Die eingeführten und entzündeten Schwefelteilchen sinken nicht zu Boden, sondern sie werden durch die Flamme und den Auftrieb getragen, so daß der gesamte. Verbrennungsraum von einem Flammenmeere ausgefüllt ist und eine absolute Ausnutzung des Sauerstoffes zur Bildung von $\mathrm{SO}_{2}$ erzielt wird, was durch das bisherige Ủberleiten von Luft über geschmolzenen Sohwefel nicht erreicht werden konnte.

Zur Röstung des Schwefelkieses werden jetzt die $\mathrm{m}$ e ch a n is c hen $R$ ös töf en immer mehr

7) Nr. 194 948; diese Z. 21, 93.1 (1908).

8) Nr. 191 596; Chem. ZentralbI. 1908, I, 314. benutzt. Von deń neuen Verbesserungsvorschlägen für diese Öfen wären folgende zu erwähnen:

Scherfenberg und Prager ${ }^{\theta}$ ) bringen pflugscharähnliche Schaufeln an den Rührarmen der Welle an, wodurch eine große Ausnutzung der Röstfläche erreicht und der Kraftverbrauch des Ofens auf ein Minimum reduziert werden soll. Der Schwefelkies wird von der Fläche abgehoben und in sehr vollkommener Weise umgewendet.

Da manche Pyrite in den 5 etagigen Öfen auf der 2. oder 3. Platte, wo die höchste Temperatur herrscht, zusammenbacken, will $K$ a uf $m$ a $n$ das Rührwerk an diesen Stellen dadurch wirksamer gestalten, daß hier die Zähne der Rührarme eine größere Schrägstellung und damit eine größere Arbeitsfläche als die übrigen erhalten.

Die Frage der zweckmäßigsten Ausgestaltung der Vorrichtungen zum Entfernen des Flugstaubes aus den Röstgasen der mechanischen Öfen ist noch nicht endgültig gelöst.

$S$ cherfenberg und Prager ${ }^{10}$ ) bauen eine neue Flugstaubkammer (Fig. 1). Diese soll bei geringerer Größe eine vollständige Abson-

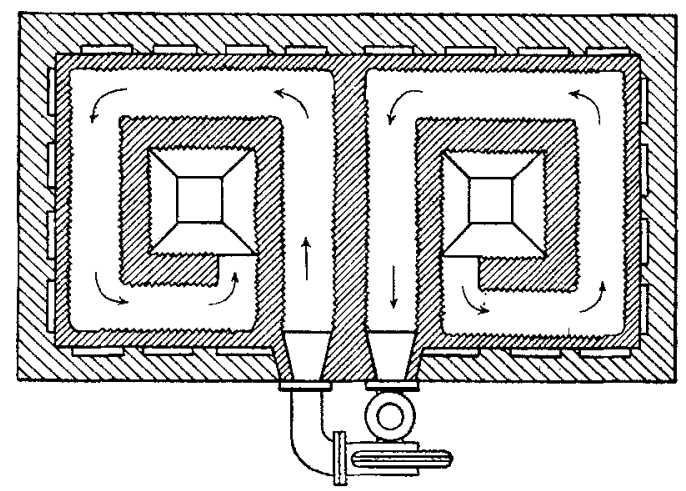

Fig. 1.

derung des Flugstaubes gewährleisten. Es wird dies dadurch erreicht, daß die inneren Wandungen der Kammer nicht, wie bisher üblich, eine glatte, sondern eine stark geriffelte Oberfläche aufweisen.

Was nun die Neuerungen an den Bleikammern selbst betrifft, so wäre zunächst ein Vorschlag von Lou is Georges Fromont B r ü s s e 11) zu erwähnen:

F r o m o n t will die Kammerwände nicht mehr glatt gestalten, sondern ihnen eine gewellte Oberfläche geben.

E u g e $n$ H ö 1 i n g baut in die Eingangs- und Úbergangsrohre der Kammern Spiralen oder spiralenförmige Segmente, um die Gase in eine rotierende Bewegung zu versetzen und dadurch eine innige Mischung zu bewirken.

Denselben Zweck will $R$ udolf $H$ e in z ${ }^{12}$ ), t e chn. Bu reau für chem. Ind., Han nover (Fig. 2), dadurch erreichen, daB er die vom Kiesofen oder Glover kommende Gasleitung teilt und in die hintereinander oder parallel ge-

9) Reusch. Chem.-Ztg. 1909, 190.

10) Nr. 203 948; Chem. Zentralbl .1908, II. 1790.

11) Nr. 191 723; diese Z. 21, 793 (1908).

12) D. P. A. Kl. $12 i$ H. $404-19$. 
schalteten Kammern durch sich gegenüberstehende Öffnungen so einleitet, daß die eintretenden Gase gegeneinander prallen und sich misehen.

Die Intensität der Leistung eines Kammersystems will $\mathrm{Hugo}$ Petersen-Wilmers dorf b. Berlin ${ }^{13}$ ) durch eine vermehrte Zufuhr von Stickoxyden steigern. Um nicht eine große Menge dünner Gloversäure eindampfen zu müssen, verwendet er neben dem üblichen System von mit $60^{\circ}$ igen Säuren berieselten Glover- und Gaylussactürmen noch ein zweites System von Glover und

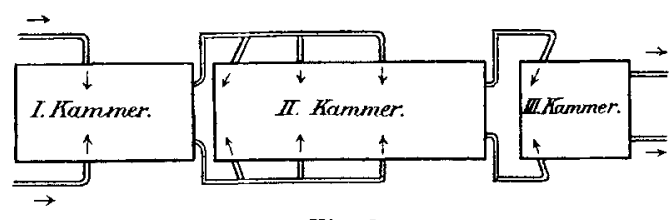

Fig. 2.

Gaylussac, die mit einer schwächeren Säure berieselt werden. Diese Säure besitzt eine solche Stärke, daß sie einerseits die niederen Stickoxyde leicht aufnehmen und anderseits dieselben, obne daß es. der Anwendung hoher Temperaturen bedarf, wieder abgeben kann. Diese Eigenschaft, welche der $60^{\circ}$ igen Säure nicht zukommt, besitzt eine Säure von $54-55^{\circ}$ Bé. Mit dieser Säure wird das zweite unter sich geschlossene System berieselt. Das Schema einer solchen Anlage wäre also:

1. Glover mit $60^{\circ}$ iger Nitrose und Kammersäure.

2. Denitrierturm mit 54-55 iger Nitrose von Gay-Lussac des II. Systems.

3. Bleikammern.

4. Gay-Lussac des II. Systems mit 54 bis $55^{\circ}$ iger Gloversäure.

5. Gay-Lussac des I. Systems mit $60^{\circ}$ iger Gloversäure.

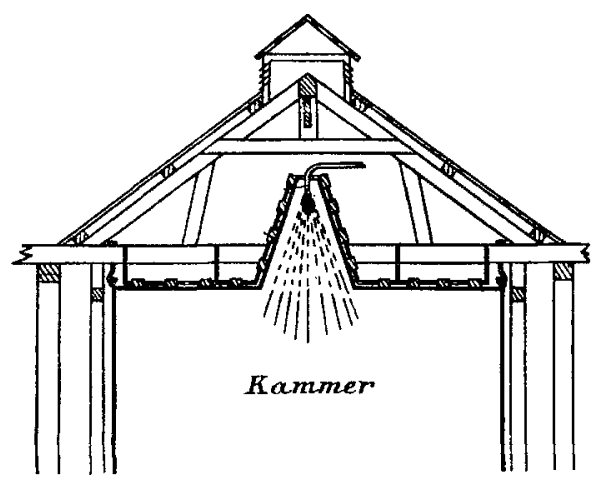

Fig. 3.

Neuerungen in der Wasserzerstäubung bringt Antonio Gaillard-Barcelona14) (Fig. 3). Er versieht die Decke der Kammern mit Aufsätzen von solcher Höhe, daß eine für die vollständige Verdampfung erforderliche Fallhöhe ohne Vergrößerung des Kammerraumes herbeigeführt wird.

13) D. P. A. Kl. $12 i$ P. 19293 ; diese Z. 21, 1235 (1908).

14) Nr. 203749 u. 205912 ; diese Z. 22, 271 u. $648(1909)$.
In einem weiteren Patente will er das Einfallen von Wassertropfen in die Bodensäure dadurch verhindern, daß er auf dem Kammerboden unter der Verstäubungsvorrichtung eine aus Steinplatten gebaute $A$ uff a $\mathrm{n} g$ w a $n$ e anbringt. Die Wanne ragt mit ihrem obersten Rand über den Spiegel der Bodensäure. Das angesammelte $\mathrm{H}_{2} \mathrm{O}$ wird nach außen abgeführt.

$\mathrm{Sch}$ erfe $\mathrm{nberg}$ und Prager ${ }^{15}$ ) bauen in die Kammern Zwischenwände, die durchbrochen sind und zweckmäßig eine geriffelte oder ähnliche Oberfläche aufweisen. $\mathrm{Zu}$ diesen $\mathrm{Zwischenwänden}$ werden nun die in der Kammerdecke befindlichen Düsen so angeordnet, daß das eingespritzte $\mathrm{H}_{2} \mathrm{O}$ auf die Seitenflächen der Querwände treffen muß. Diejenigen Teile des eingespritzten Wassers, die bis zur Berührung der Wandflächen mit den Gasen nicht in Reaktion getreten sind, werden dann von den Zwischenwänden aufgenommen, an und in denen sie langsam hinunterrieseln, um von den vorher in Reaktion getretenen durchströmenden Gasen vollständig getroffen zu werden, so daß in die Kammerschale keine verdünnende Flüssigkeit, sondern immer nur eine verstärkte Säure gelangen kann.

Aus den Endgasen will $\mathrm{H}$ a $\mathrm{n} s \mathrm{~K}$ ü h $\mathrm{n}$ e Gosla $\mathrm{r}^{16}$ ) alle $\mathrm{SO}_{2}$ durch Einwirkung des altravioletten Lichtes entfernen. In einem Turm brennen Quarz- oder Uviollampen. Die $\mathrm{SO}_{2}$ soll nun von dem $\mathrm{H}_{2} \mathrm{O}$, mit dem der Turm berieselt wird und unter Einwirkung von Luft und Licht in $\mathrm{H}_{2} \mathrm{SO}_{4}$ verwandelt werden.

Eine neue Konzentrationsvorrichtung für $\mathrm{H}_{2} \mathrm{SO}_{4}$ hat sich A n t o $\mathrm{n}$ i o G a i 11 a r d - B a reeIon a 17) patentieren lassen. Sie besteht darin, daß in einem Turme die zu konzentrierende Säure zerstäubt wird, und den herunterfallenden Säuretröpfchen heiße Feuergase entgegengeführt werden.

Schließlich wäre hier noch zu erwähnen, daß die Bemühungen von $\mathrm{Hartmann}$ und Benker, bei ihrem Konzentrationsapparat die Porzellanschalen durch solche aus Metall zu ersetzen, von Erfolg gewesen zu sein scheinen. Wenigstens garuntieren sie jetzt eine Widerstandsfähigkeit ihrer Schalen aus Neutraleisen gegen kochende Säure auf die Dauer von 12 Monaten.

Kontaktverfahren.

Hier wurden im verflossenen Jahre fast gar keine Neuerungen bekannt. Fs sind eben die verschiedenen Kontaktverfahren so gründlich ausgearbeitet, daß eine umwälzende Neuerung wohl nicht vorauszusehen ist und kleinere Verbesserungen an Apparatur werden als Betriebsgeheimnis betrachtet und nicht patentiert.

N. L. He in $\mathbf{2}^{18}$ ), Verein ig te St a t en, will eine Verbesserung des Kontaktprozesses dadurch herbeiführen, daß zunächst etwa $20 \%$ der in den Röstgasen vorhandenen $\mathrm{SO}_{2}$ nach dem Prinzip der Bleikammern in $\mathrm{H}_{2} \mathrm{SO}_{4}$ verwandelt werden. Hierdurch soll eine so weitgehende Reinigung der Röstgase von den Kontaktgiften und störenden Nebeln eintreten, daß die restlichen $80 \%$ der $\mathrm{SO}_{2}$

15) D. P. A. KJ. $12 i$ Sch. 30248.

16) Nr. 203541 ; diese $Z .22,267$ (1099).

17) Nr. 192 155; diese Z. 21, 796 (1908).

18) Reusch. Chem.-Ztg. 1909, 190. 
nach einer leicht durchführbaren, nachträglichen Reinigung in üblicher Weise in Anhydrid übergeführt werden können.

$\mathrm{Zu}$ erwähnen wäre ferner ein neuer Kontaktofen von $\mathrm{B}$ abatz-Rheingön thei m${ }^{19}$ ) (Fig.4): Die $\mathrm{SO}_{2}$-Gase durchziehen mehrere getrennte, auf der Eintritt- und Austrittseite durch Siebflächen abgeschlossene Kontakträume. Zwischen zwei Kontakträumen ist ein Kühlraum eingeschaltet. Der Kühlraum besitzt ein System von Röhren, durch welches Luft strömt. An diese Luft geben die Gase ihre überschüssige Wärme $a b$ und gelangen mit der nötigen Temperatur in den nächsten Kontaktraum. Dadurch wird eine einheitliche Temperatur in allen Kontakträumen erzielt, die Kontaktsubstanz wird vollkommen ausgenutzt und mit der vollständigen Vereinigung der Gase wird deren umzusetzende Menge erhöht.

Dr. Frederick Gardner ${ }^{20}$ ), Vereinigte Staaten, patentiert ein Verfabren zur Darstellung von $\mathrm{H}_{2} \mathrm{SO}_{4}$ nach dem Kontaktprozeß. Die das $\mathrm{SO}_{3}$ enthaltenden Kontaktgase werden auf eine mäßige Temperatur abgekühlt und entweder durch $\mathrm{H}_{2} \mathrm{O}$ oder verdünnte $\mathrm{H}_{2} \mathrm{SO}_{4}$ geleitet, oder es wird eine dieser beiden Flüssigkeiten in Dampfform in die Reaktionsgase eingeführt. Die Menge von $\mathrm{H}_{2} \mathrm{O}$ oder verdünnter $\mathrm{H}_{2} \mathrm{SO}_{4}$ muß so bemessen sein, daß die entstehenden Sprühtröpfehen von $\mathrm{H}_{2} \mathrm{SO}_{4}$ die gewïnschte Konzentration haben. Die freien $\mathrm{H}_{2} \mathrm{SO}_{4}$-Teilchen, die den Sprühregen bilden, werden von dem überschüssigen Gase durch Zentrifugieren oder durch Anwendung elektrischer Entladungen getrennt und gesammelt, die Gase wiederholt mit der Kontaktmasse in Berührung gebracht.

Neue Wege, $\mathrm{SO}_{3}$ fabrikmäßig darzustellen, geben die Patente von $R$ udolf Frank Grunewald b. Berlin'21). Durch Verbrennung von Schwefel unter einem Drucke ron 40-50 Atm. werden, wie bekannt, etwa $50 \%$ des $\mathrm{S} \mathrm{zu} \mathrm{SO}$ verbrannt. Das neue Verfahren beruht nun auf der Beobachtung, daß die Bildung von $\mathrm{SO}_{3}$ bei einem höheren Drucke, d. h. über 100 Atm. fast quantitativ verläuft. Als Ausgangsprodukt dient ein Gemisch von $\mathrm{SO}_{2}$ und überschüssigem Sauerstoff. Ein Kompressor für hohen Druck saugt ein Gemisch von $\mathrm{SO}_{2}$ und überschüssigem Sauerstoff an und komprimiert dieses Gemisch unter einem Druck über $100 \mathrm{Atm}$. Es soll nun die Bildung von $\mathrm{SO}_{3}$ glatt stattfinden. Die bei der Reaktion freiwerdende Wärme kann zur Vorwärmụng des Gasgemisches dienen. Das unter hohem Druck aus dem Kompressor ausströmende Gas wird expandiert. Dadurch wird die Temperatur so weit heruntergedrückt, daB eine Verflüssigung und schließlich ein Festwerden des $\mathrm{SO}_{3}$ erfolgt.

Dr. Hermann H ilbert-Lüt t i e h ${ }^{22}$ ) gewinnt $\mathrm{SO}_{3}$ als Nebenprodukt bei der Glasfabrikation. Es werden unter Vermeidung aller C-haltigen Zuschläge an Stelle der bisher gebräuchlichen Erden, deren Sulfate zur Zusammensetzung des Glassatzes genommen, und die beim Schmelzen freiwerdende $\mathrm{H}_{2} \mathrm{SO}_{4}$ für sich aufgefangen und kondensiert. Man arbeitet am besten in elektrischen Öfen,

19) D. P. A. Kl. 12i R. $43062 \mathrm{iv}$. 7. 9. 1908.

20) D. P. A. Kl. $12 i$ C. 16072 v. 24./12. 1908.

21) Nr. 194879 ; diese Z. 21, 796 (1908).

22) Nr. 207761 ; diese Z. 22, 648 (1909). weil sonst die Verwertung der mit sehr viel $\mathrm{CO}_{2}$ verdünnten $\mathrm{SO}_{2}$-Gase schwierig ist. So wird ein Gemisch von Abfallgips mit Sand und Alkalisulfat im elektrischen Ofen bei etwa $1250^{\circ}$ geschmolzen. Die sich bildenden $\mathrm{H}_{2} \mathrm{SO}_{4}$-Dämpfe zerfallen natürlich bei dieser Temperatur. Die $\mathrm{SO}_{2}$ wird dann nach irgend einem der bekannten Kontaktverfahren in $\mathrm{SO}_{3}$ umgewandelt.

In das Gebiet der Schwefelsäurefabrikation gehören schließlich noch die Patente von F. Benker-Chlichy ${ }^{23}$ ), Frankreich. Benker gewinnt freie $\mathrm{H}_{2} \mathrm{SO}_{4}$ aus Natriumbisulfat, indem er ein Gemisch von Bisulfat, $\mathrm{SiO}_{2}$, Sand, Silicaten unter Vermeidung der Schmelzung erhitzt.

Die Sodaindustrie, sowohl das Le B lanc-als das Solvay-Verfahren, die Fabrikationder Atzalkalien und die Sta Bfurter Industrie

haben im verflossenen Jahre keine besonderen Neuerungen gebracht.

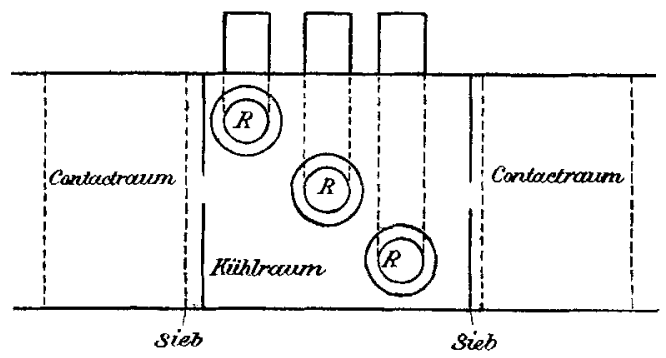

Fig. 4.

Zu erwähnen wäre ein Verfahren des V e r eins chemischer Fabriken ${ }^{24}$ ) in Mannheim zur Uberführung von $\mathrm{Na}_{2} \mathrm{~S}$ in wasserfreies $\mathrm{Na}_{2} \mathrm{CO}_{3}$ und nahezu unverdünnten $\mathrm{H}_{2} \mathrm{~S}$ durch Umsetzung mit $\mathrm{NaHCO}_{3}$. Bisher war es nicht möglich. die Zerlegung von $\mathrm{Na}_{2} \mathrm{~S}$ durch Bicarbonat in $\mathrm{Na}_{2} \mathrm{CO}_{3}+\mathrm{H}_{2} \mathrm{~S}$ quantitativ verlaufend $\mathrm{zu}$ gestalten:

$$
\mathrm{Na}_{2} \mathrm{~S}+2 \mathrm{NaHCO}_{3}=2 \mathrm{Na}_{2} \mathrm{CO}_{3}+\mathrm{H}_{2} \mathrm{~S} \text {. }
$$

Es gelingt dies aber, wenn man pulveriges $\mathrm{Na}_{2} \mathrm{~S}$ mit der nahezu äquivalenten Menge von Bicarbonat mischt und das Gemenge unter Überleiten von $\mathrm{H}_{2} \mathrm{O}$ Dampf erhitzt. Es wird ein $\mathrm{Na}_{2} \mathrm{CO}_{3}$-haltiges Produkt erhalten, das vollkommen frei von Sulfiden ist.

Die darin enthaitene, aus dem $\mathrm{Na}_{2} \mathrm{~S}$ stammende Kohle kann nach Herauslösung der Soda wieder gebraucht werden.

Das entweichende $\mathrm{H}_{2} \mathrm{~S}$-Gas ist nahezu unverdünnt und kann außer für die Darstellung von Schwefel nach dem Changeverfahren für die verschiedensten sonstigen Zwecke verwandt werden.

Der Kuriosität halber erwähne ich hier noch ein Verfahren zur Gewinnung von Soda aus $\mathrm{NaCl}$ von Claus-Nissen- Rib ber-Christi . a n i a ${ }^{25}$ ). Man unterwirft $\mathrm{NaCl}$ der Elektrolyse, wobei einerseits $\mathrm{Cl}$, und anderseits $\mathrm{NaOH}$ und $\mathrm{H}$ erhalten werden. Das gebildete $\mathrm{Cl}$ führt man in be kannter Weise z. B. unter Verwendung des entstandenen $\mathrm{H}$ in $\mathrm{HCl}$ über und nimmt diese in $\mathrm{H}_{2} \mathrm{O}$

23) Nr. 204 353; diese Z. 22, 363 (1909).

24) Nr. 194 994; diese Z. 21, 787 (1908).

25) Nr. 207 701; diese Z. 22, 644 (1909). 
auf. Mit Hilfe dieser gewonnenen $\mathrm{HCl}$ löst man Phosphate, wie Apatit u. dgl. und fügt zu der erhaltenen Lösung $\mathrm{CaCO}_{3}$ unter Umrühren hinzu. Dabei erhält man einerseits ein als vorzügliches Düngemittel dienendes Diphosphat in präcipitiertem Zustande, während anderseits sich $\mathrm{CO}_{2}$ entwickelt. Diese wird in das bei der Elektrolyse erhaltene Ätznatron geleitet und so Soda erzeugt.

\section{Sulfat und Salzs äure.}

Auch dieser Zweig der anorganischen Großindustrie hat im vergangenen Jahre nicht besonders auffallende Fortschritte zu verzeichnen. In Amerika soll der $\mathbf{L}$ u n g e - R o h r m a n n sche Plattenturm bei der Absorption der $\mathrm{HCl}$ die früheren Einrichtungen zum größten Teil verdrängt, aber auch der Ce1larius-Tourill viel Verwendung gefunden haben. Die Benutzung der Druckgasfeuerungen für Beheizung der Sulfatöfen soll dort immer, mehr zunehmen. In Deutschland führen sich mechanische Sulfatöfen langsam ein.

Von Neuerungen wäre weiter zu erwähnen: ein Verfahren zur Gewinnung von Sulfat aus B is u 1 fat von Dr. O. $\mathrm{Zahn}^{26}$ ). Z Z h n fabriziert Sulfat aus Bisulfat, indem er durch einen Zusatz voń Wasser das Bisulfat in $\mathrm{Na}_{2} \mathrm{SO}_{4}$ und $\mathrm{H}_{2} \mathrm{SO}_{4}$ verwandelt. Die freie Säure dampft er ab und calciniert das zurückbleibende Sulfat.

Für die Reinigung der Salzsäure von Arsen sind 2 Patente erteilt worden:

Der Verein chemis cher Fabriken ${ }^{27}$ ) in Mannheim entfernt das Arsen aus der Salzsäure durch innige Mischung mit Mineralölen. Das Arsen muß als Chlorür vorhanden sein. Bei einer schwachen Salzsäure leitet man gasförmige Salzsäure zur Verstärkung ein und verwandelt dabei alles Arsen in Chlorür.

Die Chemische Fabrik Grieshei m ${ }^{28}$ ) entfernt das Arsen aus der Salzsäure durch Vermisehen mit Dichlorbenzol. Hier kann das Arsen auch als Chlorid vorhanden sein. Ein Vorteil dieser Reinigungsart liegt darin, daß man das verwandte Dichlorbenzol sehr leicht völlig regenerieren kann, was bei den Teerölen nicht möglich ist.

Man vermischt das Dichlorbenzol mit der doppelten Menge Wasser, destilliert das Dichlorbenzol ab und gewinnt es so ganz arsenfrei wieder.

G. Schollmeyer-Cöthen ${ }^{29}$ ) patentiert ein ganz eigenartiges Verfahren zur Gewinnung von Salzsäure. Als Rohmaterial benutzt er $\mathrm{CaCl}_{2}$ und zersetzt dieses mit Kieselfluorwasserstoffsäure. Es entsteht nun nach der Gleichung:

$$
\mathrm{CaCl}_{2}+\mathrm{H}_{2} \mathrm{SiF}_{6}=\mathrm{CaSiF}_{6}+2 \mathrm{HCl}
$$

Salzsäure und Kieselfluorcalcium. Man dampft bis zur Trockene ein und erhitzt den Eindampfrückstand. Dabei zerlegt sich das Kieselfluorcaloium:

$$
\mathrm{CaSiF}_{6}=\mathrm{CaF}_{2}+\mathrm{SiF}_{4} \text {, }
$$

und es bildet sich neben $\mathrm{CaF}_{2}$ Siliciumfluorid, das in bekannter Weise wieder in Kieselfluorwasserstoffsäure verwandelt wird. Man gewinnt also 2/3 der verwandten Kieselfluorwasserstoffsäure wieder

26) Reusch. Chem.-Ztg. 1909, 190.

27) Nr. 184 325; diese Z. 21, 791 (1908).

28) Nr. 194864 ; diese Z. 21, 791 (1908).

29) Nr. 191 830; diese Z. 21, 790 (1908). zurück. Der Vorteil des neuen Verfahrens besteht in der Möglichkeit, billig eine hochprozentige, völlig arsen- und schwefelsäurefreie Salzsäure gewinnen zu können.

Eine Abänderung der Salzsäuregewinnung aus $\mathrm{H}$ und $\mathrm{Cl}$ bringt $\mathrm{I} s$ a i a $\mathrm{h} \mathrm{L}$ e $\mathrm{v}$ is $-\mathrm{R}$ o ber t $\mathrm{s}^{30}$ ). Um eine von Wasserstoff ganz freie Salzsäure zu erhalten, werden molekulare oder ungefähr molekulare Mengen von Chlor und Wasserstoff in der Weise zur Reaktion gebracht, daß die Gase durch besondere Rohre dem Verbrennungsofen zugeführt werden. In dem Ofen gelangen die Gase unmittelbar nach dem Zusammentreffen durch Entzündung zur Vereinigung.

Anschließend an die Sulfat- und Salzsäureindustrie wären noch einige Vorschläge für Reinigung des Steinsalzes zu erwähnen:

Balfour Fraser M c Tear 31 ) schmilat das Salz, rührt es kräftig durcheinander und läßt es in flüssigem Zustand bis zur Absonderung der Beimengungen stehen. Später verbessert er seine Reinigungsmethode dadurch, daß er das geschmolzene Salz in Behälter fließen läßt, in die ein Einsatz eingehängt ist. Der Einsatz besitzt keinen Boden, so daß er aus dem Behälter herausgenommen werden kann, ohne dabei viel von dem gereinigten Salz mitzunehmen. Wird der Einsatz vor dem Erstarren des Salzes herausgezogen, so bleibt das unreine Salz an seinen Wänden hängen.

$\mathrm{H}$ a rry $\mathrm{Te} \mathrm{e}^{32}$ ) reinigt Steinsalz dadurch, $\mathrm{da} B$ er durch das geschmolzene Salz Wasserdampf, Kohlensäure, Generatorgas unter Druck hindurchtreibt und unter Beibehaltung der Schmelztemperatur nachher die Masse absitzen läßt.

Aus unreinen Salzsolen erhält St. M a $\mathrm{r} \mathrm{h}$ ville-Paris ${ }^{33}$ ) ein reines Salz. Er läßt die Sole über geheizte Verdampfkörper fließen. An den Wandungen dieser sollen Verunreinigungen wie Gips haften bleiben, während kein Kochsalz zur Ausscheidung kommt.

Die Industrie des

\section{Chlors}

hat in dem Golds chmidtschen Verfahren ${ }^{34}$ ) zur Abscheidung des Chlors aus chlorhaltigen Gasgemischen einen Fortschritt zu verzeichnen.

Aus einer elektrolytischen Zersetzungszelle leitet man das erhaltene Chlor zunächst durch einen Trockenapparat, und zwar einen Schwefelsäureturm. Das aus dem Turm entweichende Chlor enthält $80 \mathrm{Vol} . \% \mathrm{Cl}$ und $20 \%$ angesaugte Luft und Sauerstoff, sowie Kohlensäure aus dem Anodenraum. Man leitet nun dieses auf $-20^{\circ}$ gekühlte, auf $1,3 \mathrm{Atm}$ gepreßte Chlor in auf gleich niedere Temperatur gekühltes Zinnchlorid. Hat sich so viel Chlor in diesem angehäuft, daß das Gewicht des angewandten Zinnchlorids sich verdreifacht hat, so läßt man das Gemisch in eiserne Zylinder. Sofort beginnt das Chlor energisch zu entweichen. Durch ganz gelinde Erwärmung kann der Austritt des Chlors wesentlich befördert werden.

30) Nr. 194 947; diese Z. 21, 797 (1908).

31) Nr. 206410 ; diese Z. 22, 549 (1909).

32) Nr. 206 569; diese Z. 22, 549 (1908).

33) Nr. 206 409; diese Z. 22, 549 (1909).

34.) $\mathrm{Nr}$. 206 104; diese Z. 22, 364 (1909). 
P r of. Di effen b a $\mathrm{h}^{35}$ ) schlägt vor, die Flüchtigkeit der als Kontaktsubstanz beim De a c onProzeß benutzten Kupfersalze dadurch zu verringern, daß man Doppelverbindungen mit Alkalichloriden und anderen Chloriden benutzt. Als besonders geeignet erwies sich das Doppelsalz Kupferchlorid-Chlornatrium. Es entläBt ebenso wie das reine Kupferchlorid beim Erhitzen auf höhere Temperatur die Hälfte des an Kupfer gebundenen Chlors und geht in das entsprechende Chlorürdoppelsalz über. Das letztere kann aber nun bis $550^{\circ}$ erhitzt werden, ohne daß eine Verflüchtigung eintritt. Die Verwendung dieses Doppelsalzes ermöglicht es also einerseits, bei dem bisherigen Temperaturmaximum ohne Verlust an Kupfersalz zu arbeiten, so daß die Leistungsfähigkeit der Apparate konstant bleibt, und das lästige Neuimprägnieren der Tonmasse in Wegfall kommt; andererseits gestattet sie, mit der Anfangstemperatur erheblich höher zu gehen und dadurch die Reaktionsgesch windigkeit bedeutend zu steigern.

Chlorverbindungen des Kalks und konz. Hypochloritlösungen erzeugt die $\mathrm{ch}$ emis $\mathrm{ch}$ e Fabrik Griesheim-Elektron ${ }^{36}$ ). Das Verfahren beruht auf der Beobachtung, daß bei Einleiten von Chlor in Kalkhydrat bei einer erreichten Dichte von 1,15 wasserlösliche Verbindungen des Hypochlorites mit Kalk ausfallen. Durch weiteres Behandeln mit Chlor gehen die Körper wieder in Lösung. Wird also eine Kalkmilch ron 1,15 Dichte mit einer geringen Menge Kalk versetzt und unbekümmert um das Ausfallen der basischen Verbindungen Chlor eingeleitet, bis die basischen Verbindungen sich wieder zersetzt haben, so kann man zu Chlorkalklösungen beliebiger Konzentration kommen, ja man kann die Konzentration so weit treiben, daß sich reines krystallisiertes ,unterchlorigsaures $\mathrm{Ca}^{\text {" }}$ ausscheidet.

Für Verbesserungen der Fabrikation von

\section{Salpetersäure}

sind mehrere Patente erteilt worden. Man ist vor allem bemüht, bei der Fabrikation aus Chilesalpeter eine möglichst hochkonzentrierte Säure unter möglichst geringen Verlusten zu fabrizieren. Man arbeitet heute entweder nach dem $\mathrm{V}$ a l e $\mathrm{n} \mathbf{t}$ i n e r $\mathrm{Schwarz}-\mathrm{Verfah}$ e $\mathrm{n}$ (Destillation unter vermindertem Druck) oder mit dem Uं b el schen Apparate, der einen kontinuierlichen Betrieb gestattet.

Scherfenberg und Prager ${ }^{37}$ ) bauen eine neue Salpetersäurekondensation. Durch diese Kondensationsanlage sollen die gebräuchlichen Schlangenkühler oder sonstige, im ersten Stadium der Salpetersäurekühlung übliche, mit vielen Knie- und Bogenstücken ausgerüstete Kühler ersetzt werden. Es ist hier eine an sich bekannte, aus mehreren senkrechten und parallel zueinander angeordneten Kondensationsrohren bestehende Kühlvorrichtung als Rückflußkühler unmittelbar über dem Kondensationsturm der Salpetersäureanlage angeordnet. Hierdurch werden die vom ersten Turme aufsteigenden und durch eine Spritzkappe zerstäubten

35) Nr. 197955 (jetzt zurückgenommen); diese Z. 21, 1562 (1908).

36) Nr. 195 896; diese Z. 21, 1517 (1908).

37) Nr. 202 560; diese Z. 21, 2518 (1908).

Ch. 1909 . heißesten Gase im Turm und in der Kühlvorrichtung mit dem gekühlten herabfließenden Kondensat beständig in innigste Berührung gebracht, so daß eine schnelle Kondensation bewirkt und nicht nur ein Gasüberdruck im Kühler vermieden, sondern beständig vielmehr in demselben eine Luftleere aufrecht erhalten wird, die sich auch dem darunter befindlichen Absorptionsturm mitteilt.

Hochkonzentrierte Salpetersäure fabriziert die Badische Anilin-\&Sodafabrik ${ }^{38}$ ) durch Destillation einer verdünnten Säure mit Bisulfat. So werden $1000 \mathrm{~kg}$ Bisulfat mit $200 \mathrm{~kg}$ $40^{\circ}$ iger Salpetersäure übergossen und im Vakuum destilliert. Man erhält so $75-80 \%$ der angewandten dünnen Säure als $92 \%$ ige.

Statt Chilesalpeter zersetzen die $\mathrm{Ch}$ mischen Werke von Dr. Heinrich BykCharlottenburgis) den jetzt leicht zugänglichen Kalksalpeter. Man löst den Kalksalpeter mit wenig verdünnter Salpetersäure auf, vermischt mit der berechneten Menge Schwefelsäure und trennt den Gips durch Abpressen. Man umgeht so die Destillation.

$\mathrm{Zu}$ erwähnen wäre noch die Konzentration von Salpetersäure nach einem $\mathrm{Pa}$ tente von $R$. Wolffenstein und $O$. Bötters40): Die zu konzentrierende Salpetersäure wird für sich erhitzt; die Dämpfe werden in erwärmte Vorlagen geleitet, die mit wasserfreien Nitraten beschickt sind. Die Wiederentwässerung der Nitrate geschieht durch Erhitzen im Vakuum.

Die Gewinnung von Salpetersäure oder auch Nitraten und Nitriten a $\mathbf{s} \mathbf{L} \mathbf{u} f \mathbf{t}$ stickst of $\mathrm{st}$ eht heute im Vordergrund des Interesses. Zu den bekannten Unternebmungen in Norwegen, die in guter Fortentwicklung begriffen sein sollen, sind im verflossenen Jahre in Skandinavien keine neuen hinzugetreten.

An der Ausgestaltung der bereits bewährten Verfahren für die Salpetergewinnung aus Luft wird von seiten der Techniker und Chomiker lebhaft weiter gearbeitet; das verflossene Jahr hat wieder eine große Anzahl diesbezüglicher Vorschläge zu verzeichnen. Ich kann hier nur auf die wichtigsten davon eingehen:

Apparat der B. A. S. F.41) (Fig. 5): Die vorliegende Erfindung ermöglicht insbesondere für

38) D. P. A. Kl. $12 i$ B. 44989 v. 21./5. 1908.

39) Nr. 208 143; diese Z. 22, 748 (1909).

40) Nr. 191912 (Zusatzpatent zu Nr. 189 865; diese Z. 21, 798 [1908]).

41) Nr. 201 279; diese Z. 21, 2330 (1908); vgl. auch $\mathrm{S}$ chö n h er r, diese Z. 21, 1633 (1908). 
Zwecke von Gasreaktionen die Herstellung außerordentlich langer, ständig brennender Lichtbogen, schon unter Anwendung verhältnismäßig geringer Spannungen. Zu diesem Zwecke wird z. B. eine im Innern eines Rohres A B nahe dem einen Ende A isoliert angebrachte Elektrode mit dem einen Pol, das sie umschließende Rohr mit dem anderen Pol einer Starkstromquelle verbunden und zwischen ihnen zunächst ein kleiner Lichtbogen erzeugt. Gleichzeitig wird ein Gas in zweckmäBig wirbelartiger Bewegung von $\mathrm{A}$ her an der innern Elektrode vorbei durch das Rohr geführt. Unter diesen Umständen verhindern, sobald die Erscheinung stabil geworden, die an der Wand des Rohres vorwärts strömenden Gase die Entladung gegen die der innern Elektrode benachbarten Rohrwände und gestalten die Entladung derart, daß sich im Innern des Rohres ein ständig brennender Lichtbogen bildet, welcher erst in einer sehr beträchtlichen Entfernung von der jnnern Elektrode die als zweite Elektrode dienende Rohrwand erreicht. Man kann diesen Lichtbogen z. B. schon mit der Spannung von einjgen 1000 Volt leicht eine Länge von 2 u nd mehr Meter $n$ geben und in einem einzigen solchen Lichtbogen Hunderte von Kilowatt zur Wirkung bringen. Statt das Rohr selbst, welches man im allgemeinen aus Eisen nehmen wird, als Elektrode zu benutzen, kann man den Lichtbogen auch an einer besonderen, kühlbaren Elektrode vorüberstreichen lassen, die innerhalb des Rohres angebracht ist. In diesem Falle dient das Rohr nur vorübergehend beim Entzünden des Lichtbogens als Elektrode, und man kann ihm diese Funktion vollständig nehmen, also z. B. ein nicht leitendes Rohr verwenden, wenn man durch geeignete Maßnahmen für die Entzündung und Entwicklung des Lichtbogens Sorge trägt.

Das Verfahren unterscheidet sich von den anderen bekannten wesentlich dadurch, $\mathrm{daB}$ die Gase mit dem axialen Lichtbogen, der sich durch die Reaktionsrohre bis zu vielen Metern lang hindurchstreckt, während der ganzen Dauer ihres Durchstreichens der Röhre, also viel länger, als sonst allgemein für richtig und zweckmäßig gehalten wird, in Berührung bleiben. Dennoch läßt sich nicht nur eine weitgehende Reaktion und somit größere Konzentration der behandelten Gase an den darzustellenden Umwandlungsprodukten erzielen als sonst beim Arbeiten mit großen Energiemengen im Dauerbetriebe erreichbar waren, sondern es wird auch ganz unerwarteter Weise eine wesentlich bessere Ausnutzung der elektrischen.Energie als bei den früheren Verfahren erreicht. Das Verfahren hat ferner vor allen anderen den großen Vorteil voraus, dab es mit der ejnfachsten und billigsten Apparatur, ohne bewegliche Teile, Elektromagnete oder ähnliche Hilfsmittel zu arbeiten gestattet, und daß infolge der hohen Konzentrationen der erhaltenen Gase deren Weiterverarbeitung z. B. die Absorption vereinfacht wird.

Apparate der Salpetersäureindustrie - Gesellschaft - Gelsen kirchen ${ }^{42}$ ) (Fig. 6): A sind verhältnismäBig breite, einige Zentimeter dicke Elektroden.

$B$ sind Zündschneiden, oder Zündnadeln. Sie sind mittels eines isolierenden Zwischenstïckes $\mathrm{C}$ mit einer Einstellvorrichtung D verbunden. e ist die Gasdüse, durch die das zu oxydierende Gasgemenge geblasen wird. Die Düse ist vorteilhaft schlitzförmig ausgebildet.

Die Zündschneiden werden in einem Schlitze oder Kanal durch die Elektroden geführt. Da sie infolge ihrer geringen Stärke schneller abbrennen als die feststehenden Elektroden, so müssen sie von Zeit zu Zeit auf die gew inschte Entfernung eingestellt werden. Die Einstellung richtet sich nach der Energiemenge, die von der Flamme bearbeitet werden soll, da man ja bekanntlich durch den Ab-

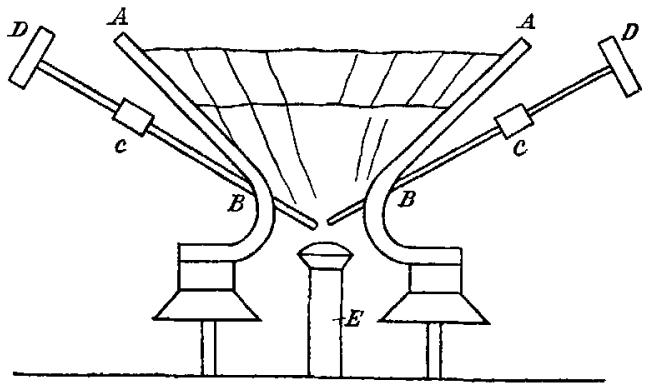

Fig 6.

stand der Spitzen die Spannung beliebig regulieren kann. Der zwischen den beiden Zündstiften erzeugte kleine Bogen wird durch den Gasstrahl den Elektroden zugeblasen und fächerförmig ausgebreitet.

Ein anderer Apparat der salpeter. $s$ äuregesells ch a $\mathrm{t}^{43}$ ) ist folgender:

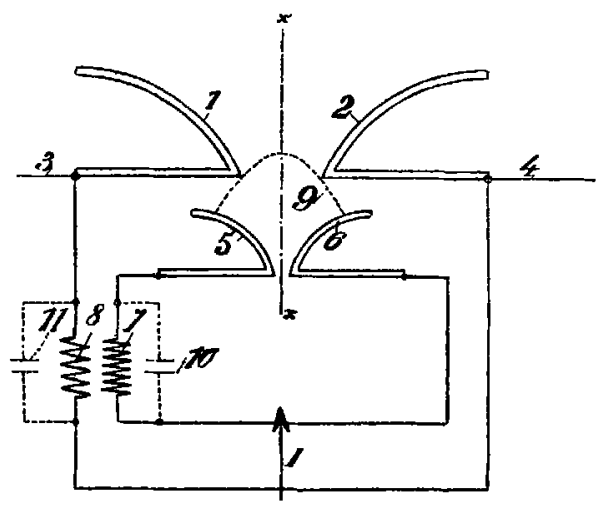

Fig. 7.

Die beiden Elektroden 1 und 2, Fig. 7, der Hörnerflammenstrecke stehen mit ihren unteren Spitzen so weit voneinander entfernt, daß eine Zündung durch die Normalspannung des durch die Leitungen 3 und 4 zugeführten Stromes ausgeschlossen ist. Unterhalb dieser Flammenstrecke ist eine zweite so angeordnet, daß beide eine gemeinschaftliche Mittelachse $\times \times$ haben, und daß die Hörnerelektroden 1 und 2 und 5 und 6 in einer Ebene liegen. Die Elektroden 5 und $b$ der zweiten Flammenstrecke sind mit den Sekundärwicklungen 7 eines Transformators, beispielsweise eines solchen mit dem U̇bersetzungsverhältnis $1: 30$ verbunden, während die

42) Nr. 198 241; diese Z. 21, 1562 (1908).

43) Nr. 205 464; diese Z. 22, 498 (1909). 
Primärwicklungen 8 des Transformators in der in der Zeichnung angegebenen Weise parallel geschaltet sind.

Führt nun z. B. die Hauptstromleitung Wechselstrom von 50 Perioden in der Sekunde und 2000 Volt Spannung, so wird bei Beginn einer jeden halben Periode die Sekundärflammenstrecke, die eine entsprechende Elektrodendistanz erhält, sehr früh zünden. Die große Luftgeschwindigkeit in dem die ganze Vorrichtung umschließenden Rohre bewirkt, daß die so eingeleitete Entladung der Sekundärflammenstrecke nach Art des punktiert eingezeichneten Bogens der Hauptflammenstrecke zugeblasen wird, so daß auch diese entzündet wird.

Apparat von Fritz Spitzer-Essen a. $R \mathbf{u h r} \mathbf{r}^{44}$ ) (Fig. 8). Der zwischen den Elektroden $A$ und $B$ überspringende Lichtbogen besitzt bei $C$ seine heißeste Zone, während die äußere Zone D kühler ist. Diese kühlere Zone wird von einem gekühlten engen Rohre, das dicht bis in die heißeste Zone reicht, durchsetzt. Um ein Zurückweichen des Flammenbogens vor dem gekühlten Rohre zu ver-
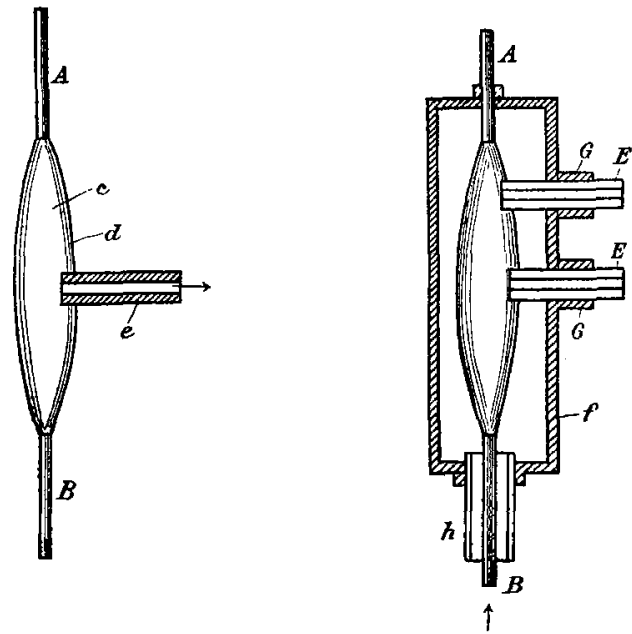

Fig. 8.

hindern, umgibt man den zwischen den vertikalen Elektroden brennenden Flammenbogen mit einem nicht zu engen Rohre F. Dieses Rohr kann zweckmäßig auch gekühlt werden. Ein Ausweichen des Flammenbogens vor dem Absaugrohr ist hierdurch ausgeschlossen, da die den Bogen umgebende gekühlte Rohrwand eine Annäherung desselben nicht gestattet. Man erhält so ein vollkommen ruhiges Brennen des Flammenbogens. Die Rohrwand ist vorteilhaft mit mehreren Ansätzen G versehen, durch welche die in einen gemeinsamen Gaskanal mündenden Absaugröhren $\mathbf{E}$ geführt sind. Die der Einwirkung des Flammenbogens auszusetzende Luft wird durcb das die Elek trode $B$ umgebende Rohr H geführt und kühlt hierbei die Elektrode.

Der Apparat von Dr. Demetrio Helbig - Rom ${ }^{45}$ ) (Fig. 9) beruht auf folgendem Prinzip: $E_{1}$ und $E_{2}$ sind Elek troden aus gewöhnlichem Material, z. B. für stärkere Ströme wassergekühlte

44) D. P. A. Kl. $12 i$ S. 23487 v. 2./7. 1908.

45) Nr. 189864 (D. P. A. Kl. $12 i$ H. 40506 v. 14./5. 1908); diese Z. 21, 5்่6 (1908).
Kupferelektroden. Sobald diese auf eine entsprechende Spannung eingestellt sind, erfolgt zwischen den Elektroden auf bekannte Weise die Entladung in Form einer zwischen den Elektroden dauernd brennenden Flamme. Wenṇ nun aber durch zwei einander gegenüberliegende Düsen ein genügend starker Luftstrom gegen diesen Bogen geschleudert wird, verwandelt sich der Bogen in zwei flache, breite Flammengarben, die, sich den Elektroden entlang ausbreitend, in einer zur Achse der Düse senkrecht liegenden Ebene sich entwickeln.

Beim Apparat von Ernst $M$ arquard Karow b. Berlin und Karl Warth. Charlotten burger) (Fig. 10) geschieht das Einleiten des Lichtbogens ohne besondere Hilfsmittel. Hängt man die Elektroden $\mathbf{E}$ an den oberen Enden beweglich oder federnd auf, parallel oder konvergierend, so werden die Elektroden vor dem Überspringen des Lichtbogens entgegengesetzt statisch geladen und werden sich deshalb gegenseitig anziehen. Infolge der Anziehung der entgegengesetzten Ladungen werden die Elektroden an den untersten Enden sich

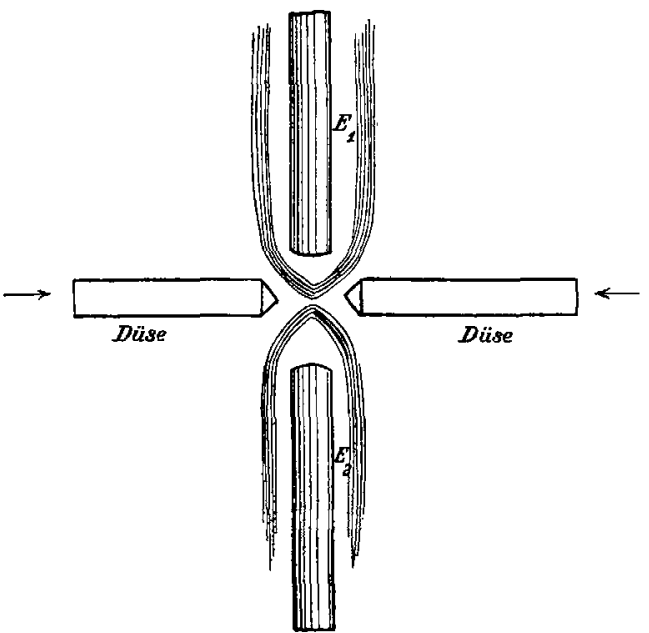

Fig. 9.

am stärksten anziehen und so weit einander nähern, daß bei Anwendung von genügend hohen Spannungen ein U̇berspringen des Funkens eintreten kann, bevor vollständige Berührung der Elektroden stattgefunden hat, Kurzschluß und Aneinanderschmelzen der Elektroden werden auf diese Weise verhindert. Der Lichtbogen wandert in die Höhe, während die Elektroden in die Ruhelage zurückkehren. Ist der Lichtbogen oben angekommen und wieder erloschen, so wiederholt sich das beschriebene Phänomen. Durch diese Anordnung ist es möglich geworden, kontinuierlich wandernde Lichtbögen in rascher Aufeinanderfolge zwischen solchen Elektroden zu erzeugen. Es ist zweckmäßig an dem untern Ende der einen Elektrode ein kleines isolierendes Distanzstück angebracht, so daß der zum Uberspringen des Lichtbogens nötige kleinste Elektrodenabstand mechanisch fixiert ist. Das Überschreiten der. Rubelage der rückkehrenden Elektroden kann gleichfalls durch entsprechend angebrachte Isolierstücke verhindert werden.

46) Nr, 200 332; diese Z. 21, 2176 (1908). 
Bei den bisher beschriebenen Apparaten diente lediglich Luft oder Gemische von Sauerstoff und Luft als Ausgangsmaterial für die zu gewinnenden Stickoxyde.

Matthias Stoll-Berlin ${ }^{47}$ ) patentiert ein Verfahren zur Herstellung von Stickoxyden durch Verbrennung von Stickstoff in einer durch Brennstoff-Sauerstoffgemisch erzeugten Flamme.

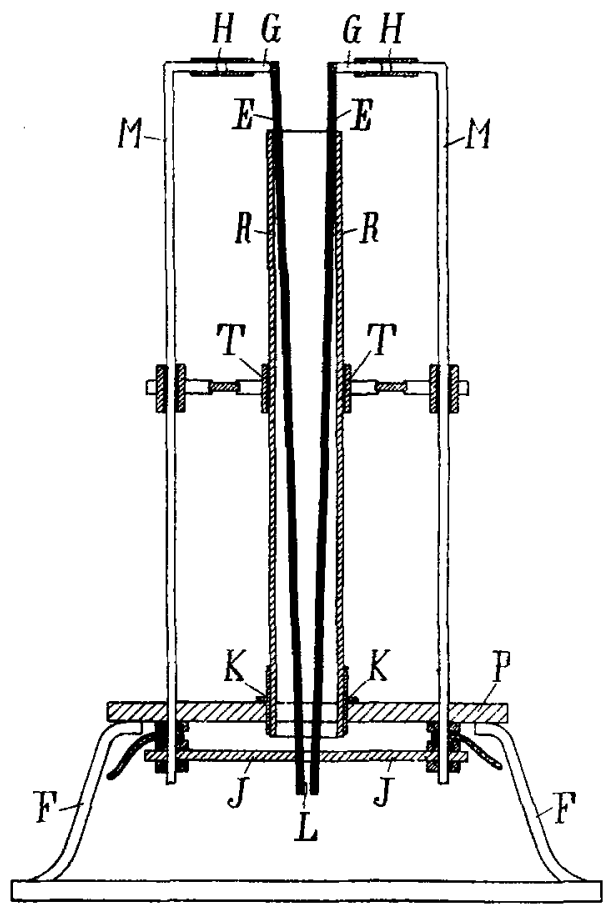

Fig. 10.

Die zur Verbrennung nötige Luft oder auch Sauerstoff werden in dem Utberhitzer U (Fig. 11) vorgewärmt und durch das Rohr d der Mischdüse C zugeführt, während die verdampften Kohlenwasser-

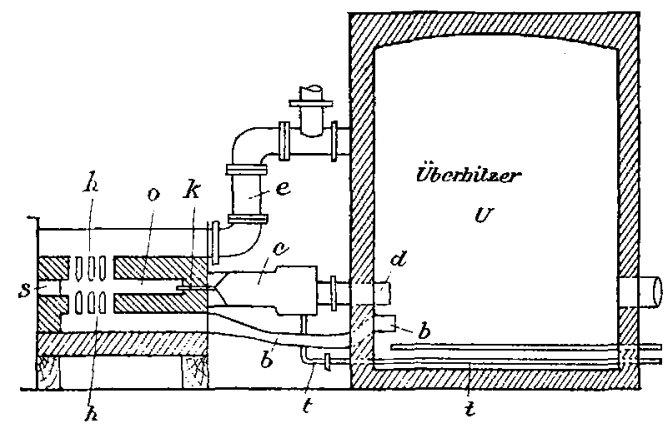

Fig. 11.

stoffe durch das Rohr t ebenfalls in die Mischdüse gelangen. Dort werden die Kohlenwasserstoffe mit der Luft derart gemischt, daß ein gleichmäßig carburiertes Gasgemisch entsteht, dessen sämtlicher Kohlenstoff zu Kohlensäure verbrennt. Die Mischdüse $\mathrm{C}$ endigt in einem Schlitzbrenner $\mathbf{K}$, welcher

47) D. P. A. Kl. $12 i$ St. 12445 v. 26./10. 1908. die Flamme im Ofenraum scheibenartig ausbreitet.

Sobald die Flamme gleichmäBig ist, was man durch das Schauloch $\mathrm{S}$ sieht, und der Ofen die nötige hohe Temperatur erreicht hat, wird durch das Rohr b überhitzter Sauerstoff oder ein sauerstoffreiches Gasgemisch eingeblasen, welches durch die Öffnungen $h$ in den Ofen gelangt, hier die scheibenförmig ausgebreitete Flamme passiert und oxydiert wird. Das aus Kohlensäure, Stickstoff, Stickoxyd und Untersalpetersäure bestehende Gasgemisch gelangt auf irgendeine praktische Weise zur Absorption.

Ein ähnliches Verfahren, bei dem außer Sauerstoff und Stickstoff noch ein gasförmiger, flüssiger oder pulverförmiger Brennstoff verwandt wird, patentiert Oskar Brïnler-Leipzig48) (Fig. 12). A ist ein geschlossener, druckwiderstandsfähiger Behälter. Derselbe ist teilweise mit Wasser angefüllt. b ist ein Wasserspeiserolhr, c ist das $\mathrm{Ab}$ -

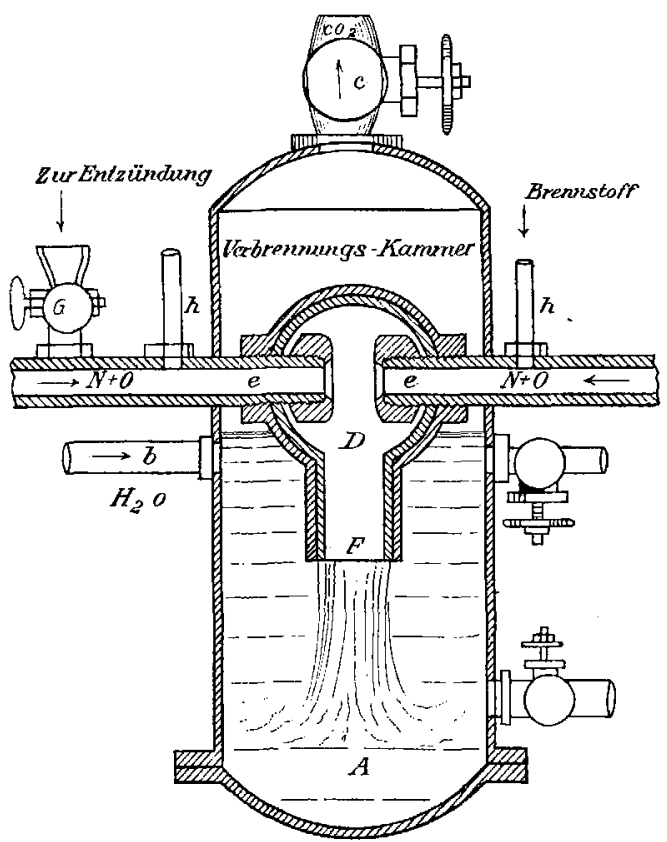

Fig. 12.

zugsrohr für verbrannte Gase, hauptsächlich Kohlensäure, e sind Zuflußrohre für Sauerstoff, dem schon ein genügendes Quantum Stickstoff beigemengt ist. $\mathrm{h}$ sind Brennstoffleitungen. $\mathrm{D}$ ist eine mit feuerfestem Chamotte oder Magnesia ausgestattete Verbrennungskammer mit einer Mündung F. G ist ein Einfüllhahn, welcher zur Entzündung bei Einleiten des Verfahrens benutzt werden kann.

Der Apparat arbeitet folgendermaßen: Das durch die Rohre e zugeleitete Gasgemenge von Sauerstoff und Stickstoff mischt sich mit dem durch die Rohre $h$ eintretenden Brennstoff. Als solcher dient in der Regel Petroleum, doch kann auch Kohlenstaub, Leuchtgas oder ein anderer flüssiger Brennstoff als Petroleum Verwendung finden.

Die Rohre e sind in den Ofen hinein verlängert eingeführt, damit das Temperaturmaximum in

48) Nr. 205538 ; diese Z. 22, 168 (1909). 
seinem Zentrum entwickelt wird und die Ofenwandungen dem Einfluß der höchsten Hitze nicht ausgesetzt und geschont werden.

Vor Inbetriebsetzung des Apparates, also solange noch kein Druck in demselben herrscht, öffnet man den Hahn $G$ und gieBt durch ihn etwas Benzin oder Spiritus ein. Diese Zündflüssigkeit wird mit einem Streichholz angezündet. Hierauf läßt man durch die Rohre $h$ Brennstoff und durch die Rohre e ein Gemisch von Stickstoff und Sauerstoff einströmen. Da die brennende Spiritus- oder Benzinflamme sich nach innen fortpflanzt, entzündet. sich das Gemisch, welches in der Verbrennungskammer D die höchste Temperatur erreicht.

Sofort nach dem Entzünden des Gemisches ist der Hahn D zu schließen. Die Verbrennungskammer ist so berechnet, und die Geschwindigkeit der durchströmenden Gase so bemessen, daß die Gase noch flammend in Form einer Stichflamme durch die Mündung $\mathrm{F}$ ins Wasser eindringen. Sobald die Temperatur innerhalb der Mischungskammer ihr Maximum erreicht hat, bilden sich Stickoxyde. Dringt die Flamme in das Wasser ein, so herrschen in ihr alle Temperaturen zwischen $2500^{\circ}$ im Zentrum und ca. $600^{\circ}$ an der Peripherie der Flamme. Sämtliche Moleküle von Stickoxyd durchlaufen in raschester Zeit diese sinkende Temperaturskala und verbinden sich mit dem noch zur Verfügung stehenden Sauerstoff zu höheren Stickoxyden, welche bei Berührung mit. Wasser Salpetersäure geben.

Sonstige Verbesserungen in Durchführung der Stickstoffaktivierung bringen die Patente der Westdeuts chen Thomas-Phosphat werke G. m. b. H. - Berlin ${ }^{49}$ ). Sie erreicht eine Steigerung der Ausbeute dadurch, daß in dem Entladungsraum ein geringerer als der Atmosphärendruck erzeugt wird. Dieser geringere Druck ruft nämlich eine sehr wesentliche Verminderung des Widerstandes der Entladungsstrecke und damit eine Vergrößerung der Flamme hervor, welche wieder eine bedeutend höhere Ausbeute im Gefolge hat. Dasselbe Ziel wollen die Thomasphosphatwerke durch eine Vorwärmung des Gasgemisches auf $1000-2000^{\circ}$ erreichen. Dadurch wird die Leitfähigkeit besser, und eine Bildung von Ozon vermieden, da dieses sich bereits bei $350^{\circ}$ zersetzt. Vor allem wird aber an Energie gespart, da jetzt nicht mehr ein Teil derselben zur Vorwärmung des Gases auf die nötige Reaktionstemperatur verloren geht.

Wichtig ist bei all den verschiedenen Verfahren eine möglichst rasche Abkühlung der Reaktionsprodukte.

Max Platsch-Frankfurt a. M.50) will eine rationelle Kühlung erreichen, wenn er als Kühlmittel staubförmige, feste, vorzugsweise sauerstoffabspaltende Substanzen, wie Superoxyde, verwendet. Diese führt er in die den Lichtbogen verlassenden Reaktionsgase ein. Die Superoxyde haben ein sehr großes Wärmeabsorptionsvermögen und kühlen die Gase stark ab. Außerdem verdünnen sie die Gase nicht und sind aus diesen leicht abzuscheiden.

Ein anderes Verfahren zur plötzlichen Abkühlung der heißen Gase ist Kunheim \& Co. -

49) D. P. A. Kl. $12 i$ W. 23711 v. 4./6. 1908.

50) Nr. 200 138; diese Z. 21, 1850 (1908).
$\mathrm{N}$ i ed ers ch ö new e i d e ${ }^{51}$ ) patentiert worden. Es besteht darin, daß die Gase durch das dicht an der Flamme vorbeiströmende Kühlmittel, welches sowohl gasförmiger, wie flüssiger Natur sein kann, zunächst angesaugt werden, so daß sie den Kühlstrahl umhüllen und mit diesem dann zusammen eine fächerförmige Ausbreitung erfahren. Es entstehen hierbei zwei dünne Schichten, deren eine vom Kühlmittel und deren andere von den zu kühlenden Gasen gebildet werden. Den Wärmeaustausch zwischen beiden erfolgt dann um so schneller, je geringer die Dicke der Schichten ist.

Als Kühlmittel können beispielsweise Verwendung finden: Luft, Wasserdampf oder ein Gemisch von beiden. Die flache Ausbreitung des Kühlmittels kann auf verschiedene Weise erfolgen. So kann z. B. die Düse, aus der das Kühlgas tritt, die Gestalt eines gewöhnlichen Schlitzbrenners haben. Die Reaktionsflamme wäre dann seitlich von dem Kühlstrahl dicht hinter der Düse anzuordnen. Eine andere Vorrichtung habe ich hier schematisch skizziert (Fig. 13):

Es finden hier zwei Kühlstrahlen Verwendung,

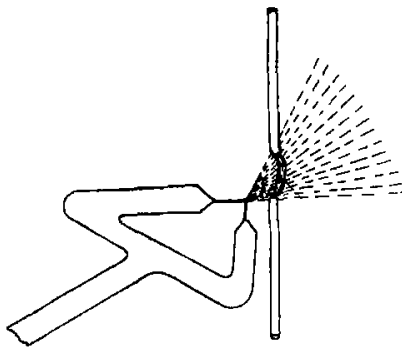

Fig. 13.

die den beiden Düsen entströmen und aufeinanderstoßen.

Was Neuerungen in der Absorption der Reaktionsgase betrifft, so wäre hier das A b sorp. tionsverfahren der Norsk Hydro. elektrisk-K vaelstof-AktieselskabKristiania zu erwähnen. Dieses benutzt als Absorptionsmittel für die nitrosen Gase ein Metallcyanamid, vor allem Calciumcyanamid. Es soll hierbei ein vollkommen haltbares Produkt gebildet werden, das aus einer Mischung von Calcium. nitrat und $\mathrm{NH}_{4} \mathrm{NO}_{3}$ besteht und ein wertvolles Düngemittel ist.

$$
\begin{gathered}
\mathrm{Ca}(\mathrm{CN})_{2}+2 \mathrm{H}_{2} \mathrm{O}+\underset{2 \mathrm{NH}_{4} \mathrm{NO}_{3}}{4} \underset{\mathrm{HNO}}{2}=\mathrm{Ca}\left(\mathrm{NO}_{3}\right)_{2}+\mathrm{CO}_{2}+
\end{gathered}
$$

In neuester Zeit $h$ at di e B. A. S. F.52) ein Verfahren zum Patent eingereicht, nach dem es möglich sein soll, aus den Reaktionsgasen direkt feste, technisch nitritfreie Nitrate zu gewinnen. Es besteht darin, daß man durch Einwirkung von Wasserdampf auf die zu absorbierenden Gase die darin enthaltenen Stickoxyde in Salpetersäure überführt, wobei es nicht von Belang ist, ob bei der betreffenden Temperatur ein Teil der Salpetersäure in bekannter Weise dissoziiert bleibt, und die so behandelten Gase von den festen Oxyden, Hydroxyden

61) Nr. 205774 ; diese Z, 22, 358 (1909).

62) D. P. A. Kl, I2i B. 50724 v. 4./2. 1909. 
oder Carbonaten der Alkalien oder Erdalkalien absorbieren läßt. Beim Zusammentreffen mit diesen Mitteln tritt eine sehr lebhafte Absorption des Salpetersäuredampfes ein und der dissoziierte Teil dieses Dampfes vermag dadurch sofort wieder neue Mengen von Salpetersäure zu bilden, so daß selbst bei höheren Temperaturen, z. B. bei $250^{\circ}$, bei denen nur ein verhältnismäßig geringer Teil der Salpetersäure in undissoziiertem Zustande beständig ist, eine überaus rasche Absorption der gesamten Salpetersäure erfolgt. Dadurch wird zugleich die Temperatur des Absorptionsmittels so. hoch gehalten, daß das bei dem Verfahren eingeführte und bei der Salzbildung wieder freiwerdende Wasser in Dampfform entweicht. Auf diese Weise erhält man praktisch nur aus Nitrat bestehende feste Produkte, während bekanntlich bei der Absorption von nitrosen Gasen ohne Wasserzufuhr Gemische von Nitrat und Nitrit und zwar z. B. bei $250^{\circ}$ etwa äquimolekulare Mengen beider Salze entstehen.

Das wären die wichtigsten Fortschritte, welche die junge elektrische Salpeterindustrie im verflossenen Jahre zu verzeichnen hatte.

Im Anschluß daran wäre hier noch ein Verfahren zur Darstellung von Stickstoffdioxyd von $\mathrm{P}$ a u l W i n a n d - K ö $1 \mathbf{n}^{53}$ ) zu erwähnen.

Dieses Verfahren beruht auf 2 Reaktionen:

$$
\begin{gathered}
\text { 1. } 2 \mathrm{KNO}_{2}+\mathrm{H}_{2} \mathrm{SO}_{4}=\mathrm{K}_{2} \mathrm{SO}_{4}+\mathrm{NO}+\mathrm{NO}_{2} \\
\quad+\mathrm{H}_{2} \mathrm{O} . \mathrm{NO} . \\
\text { 2. } \mathrm{NO}+\mathrm{NO}_{2}+\mathrm{N}_{2} \mathrm{O}_{5}=4 \mathrm{NO}_{2} .
\end{gathered}
$$

Praktisch wird die Reaktion so ausgeführt, daß man äquimolekulare Mengen von Natriumnitrit und -nitrat zusammenschmilzt und mit Oleum von $45 \%$ $\mathrm{SO}_{3}$ umsetzt:

$\mathrm{NaNO}_{3}+\mathrm{NaNO}_{2}+\mathrm{H}_{2} \mathrm{~S}_{2} \mathrm{O}_{7}=2 \mathrm{NaHSO}_{4}+2 \mathrm{NO}_{2}$. (Schlus folgt.)

\section{Fortschritte der organischen Chemie im Jahre 1908.}

\author{
Von E. Wedekind-Tübingen.
}

(Schluß von S. 1360.

4. Heterocyclische Verbindungen.

Die im Berichtsjahr erschienenen Arbeiten aus diesem Gebiete sind so zahlreich, daß ich mich mit Rücksicht auf den zur Verfügung stehenden Raum auf einen ganz kurz gefaßten Ủberblick beschränken muB.

Fünfgliederige Ringsysteme: v. Kostaniecki und V. L a m pe ${ }^{274}$ ) haben mit Studien in der $\mathrm{Cumarang} r \mathrm{uppe}$ begonnen und das $5-\mathrm{M}$ e th oxy-2-meth y lcumaran<smiles>COC1CCC2C(C)CC2O1</smiles>

synthetisiert. Homologe des $\mathrm{Cum}$ aranons bereiteten K. F ries und G. Fin $\mathbf{~ k ~}^{275}$ ); durch

53) Nr. 193 696; diese Z. 21, 412 (1908).

274) Berl. Berichte 41, 1335.

275) a. a. O. 41, 4271.
Oxydation derselben entstehen gelbe bis rote Körper. Durch Kondensation von 4-Methyleumaranon mit 4-Methyldiketocumaran entsteht der 1,2 - B is [4-methylcumaran]-indigo

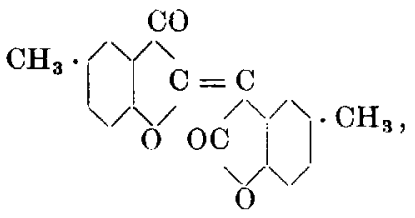

ein orangegefärbtes Sauerstoffisologes des Dimethylindirubins. Diese und ähnliche indigoide Verbindungen mit dem Cumaranonrest scheinen nicht verküpbar zu sein ${ }^{276}$ ).

Das Thionaphthen<smiles>CCC1CCCCC1S</smiles>

die Muttersubstanz des Thioindigorots, haben A. Bezdrik, P. Fried länder und P. K ö nige r 277) durch Reduktion des synthetischen Oxythionaphthens dargestellt; es ist eine krystallinische, bei $32^{\circ}$ schmelzende Masse, welche wie unreines Naphthalin riecht und sich in konz. Schwefelsäure mit kirschroter Farbe löst; von den zahlreichen Derivaten sei erwähnt das 2,3-Th i o naphthenchinon

$$
\mathrm{C}_{6} \mathrm{H}_{4} \stackrel{\mathrm{CO}}{\mathrm{CO}}
$$

das Analogon des Isatins ist; es liefert z. B. mit oPhenylendiamin Chinoxalinderivate. Thionaphthenderivate wurden auch aus Styrolverbindungen und Thionylchlorid synthetisiert278). Eine neue Synthese des $\mathrm{Th}$ i o phthens<smiles>c1cc2ccsc2s1</smiles>

erreichte G. Ca pell e $^{279}$ ) durch Einwirkung von Schwefel auf Acetylen.

Das natürliche Ox y p y r o l in (Oxypyrrolidin$\alpha$-carbonsäure) kann das Hydroxyl nur in $\gamma$ - oder $\beta$-Stellung enthalten ${ }^{280}$ ). Die Phenylhydrazone der einfachen, gesättigten hydroaromatischen Ringketone können nach W. B ors ch e ${ }^{281}$ ) im Sinne der Fis oherschen Indolsynthesen in Tetrahydrocarbazolderivate übergeführt werden, welche einerseits die Hexahydroderivate und andererseits durch Wasserstoffabspaltung die eigentlichen Carbazole liefern. Die P y r a $\mathrm{z}$ ol g r u p p e hat wieder

276) K. Fries u. G. Fin k, a. a. O. 41, 4284. 277) a. a. O. 41, 227.

278) G. B a r g e r u. A. J. Ew in s, J. chem. soc. 93,2086 .

279) Bll. Soc. chim. [4] 3, 150 .

280) H. Le uchs u. H. Felser, Berl. Berichte 41, 1726.

281) Liebigs Ann. 359, 49. 